Vol 11, Issue 10, 2018

\title{
COMPARATIVE ESTIMATION OF SALIVARY TOTAL ANTIOXIDANT CAPACITY IN PERIODONTAL HEALTH AND CHRONIC PERIODONTITIS - A PILOT STUDY
}

\author{
U RAGHAVENDRA ${ }^{1}$, ANUPAMA RAO ${ }^{2 *}$, JYOTHI D'SOUZA ${ }^{1}$, VINITHA RAMANATH PAI ${ }^{1}$, SINDHU NAIR ${ }^{1}$, \\ VIJAYA KUMAR ${ }^{2}$, BHUVANESH SUKHLAL KALAL ${ }^{1}$
}

\begin{abstract}
${ }^{1}$ Department of Biochemistry, Yenepoya Medical College, Yenepoya (Deemed to be University), Mangalore, Karnataka, India. ${ }^{2}$ Department of Periodontology, Yenepoya Dental College, Yenepoya (Deemed to be University), Mangalore, Karnataka, India.

Email: dranuperio@gmail.com
\end{abstract}

Received: 06 August 2018, Revised and Accepted: 31 August 2018

\begin{abstract}
Objective: Gram-negative bacteria provoke polymorphonuclear leukocyte (PMN) to release reactive oxygen species in chronic periodontitis (CP). Inability to maintain a balance between oxidative stress and antioxidant levels makes patients more susceptible to periodontal disease. The present study aims to estimate and compare salivary total antioxidant capacity (TAOC) in subjects with clinically healthy periodontium and patients with CP.

Methods: After fulfilling the selection criteria, a total of 20 subjects (10 with clinically healthy periodontium and 10 with CP) were subjected to unstimulated salivary sample collection for biochemical estimation of TAOC by spectrophotometric assay using Kovacevic method. Analysis of data was done with unpaired student t-test, using SPSS version 22 statistical program.
\end{abstract}

Results: Salivary TAOC was significantly higher in subjects with clinically healthy periodontium compared to CP patients. It was statistically significant $(\mathrm{p}<0.001)$.

Conclusion: This study indicated increased levels of salivary TAOC in patients with CP compared to clinically healthy periodontium. Alteration in defensive antioxidant status could be a risk factor in the progression of periodontal disease.

Keywords: Total antioxidant capacity, Chronic periodontitis, Polymorphonuclear leukocyte, Reactive oxygen species, Free radicals, Oxidative stress, Antioxidants.

(C) 2018 The Authors. Published by Innovare Academic Sciences Pvt Ltd. This is an open access article under the CC BY license (http://creativecommons. org/licenses/by/4. 0/) DOI: http://dx.doi.org/10.22159/ajpcr.2018.v11i10.28409

\section{INTRODUCTION}

Chronic periodontitis (CP) is an inflammatory disease of microbial origin with progressing destruction of supporting structures of the teeth and is the most prevalent cause of tooth loss worldwide [1]. It is initiated by the complex interaction of periodontopathogens and a susceptible host. The tissue destruction mainly occurs in individuals with hyperinflammatory response to the specific colonizing bacteria [2]. Host-microbial response generates oxidative stress leading to the increased production of reactive oxygen species (ROS), which are detrimental to periodontal health. In all the chronic inflammatory diseases production of ROS is inevitable. ROS are released from numerous sources such as the bacteria themselves, or as a consequence of the immune response due to infiltration of polymorphonuclear leukocytes (PMN) [3]. This, in turn, releases cytokines and proinflammatory mediators (interleukin [IL]-1 $\alpha$, IL-1 $\beta$, IL-6, IL-8, and tumor necrosis factor- $\alpha$ ) causing exaggeration of an inflammatory process.

Human body unfolds definite defense and repair mechanisms essentially to arrest the accumulation of oxidatively damaging toxic molecules such as $\mathrm{H}_{2} \mathrm{O}_{2}$, $\mathrm{HOCL}$, and $\mathrm{OH}^{-}$. This is achieved through antioxidant defense mechanisms (nonenzymatic and enzymatic antioxidants). The antioxidant defense mechanism aids in scavenging the free oxygen radicals and thus prevents their harmful sequela on the host by modulating inflammatory response [4].

Total antioxidant capacity (TAOC) in saliva assays is useful in giving a quantitative measure of the capacity of biological systems to withstand oxidative attack. They also throw light on the various biomolecular interactions among individual antioxidant species.
There are various reports suggesting a reduction in the TAOC in serum and gingival crevicular fluid in $\mathrm{CP}[5,6]$. The results in various studies are not able to establish the correlation between salivary TAOC in health and CP. Conflicting results of different studies about the importance of $\mathrm{AO}$ and oxidative stress levels in prevention, initiation, progression, and treatment of periodontal diseases exist in the literature [7]. The present study aims to estimate and compare salivary TAOC in health and CP.

\section{METHODS}

A total of 20 systemically healthy subjects reporting to the routine outpatient department of periodontics, were recruited in a single centered, case-control study after obtaining informed consent from them. Ethical clearance was obtained from the Institutional Ethical Committee before the study. Confidentiality of the data was maintained throughout the study period. The study group was divided into two groups.

Group I: 10 subjects with healthy periodontium

Group II: 10 subjects with CP.

\section{Inclusion criteria}

Male and female subjects within the age group of 30-50 years and with a minimum of 20 scorable teeth were included in the study. Group I included subjects with healthy periodontium, who showed no signs of periodontal disease as determined by the absence of the evidence of interproximal (CAL $\leq 1 \mathrm{~mm}$ ), no $\mathrm{PD}>3 \mathrm{~mm}$ at any sites, wholemouth bleeding scores $<10 \%$, and had no clinical signs of gingival inflammation. Group I included subjects with CP according to American Academy of Periodontology -1999 [8] as two or more tooth sites with $\mathrm{PD} \geq 4 \mathrm{~mm}$ or CAL of $4 \mathrm{~mm}$ that bled on probing. 


\section{Exclusion criteria}

Subject with a history of nonsteroidal anti-inflammatory drugs or antimicrobial drugs, mouthwashes, or vitamin/antioxidant supplements within a 3 month period before the study commences. Subjects with special dietary requirements, history of medication which influences antioxidant status, history of periodontal therapy in the previous 6 months, pregnant and lactating women, and subject with habits such as smoking, tobacco chewing, and alcoholics were excluded from the study.

Before study, ethical clearance was obtained from the Institution's Ethics Committee (Ref. No. YUEC/2015/045), and informed consent was obtained from all the study subjects.

After enrolment, a total of 20 subjects ( 10 with CP and 10 with clinically healthy periodontium) who fulfill the selection criteria were subjected to unstimulated salivary sample collection for biochemical estimation of TAOC.

\section{Collection of saliva}

Subjects were asked not to eat or drink $1 \mathrm{~h}$ before the sample collection. $2 \mathrm{~mL}$ of unstimulated whole saliva was collected by expectorating into disposable tubes before clinical measurements into a sterile container. Collected sample was centrifuged at $3000 \mathrm{rpm}$ for $15 \mathrm{~min}$, and the supernatant was stored in small aliquots at $-20^{\circ} \mathrm{C}$ until the biochemical analysis [9]. The biochemical analysis of TAOC was done using Koracevic's method [10].

\section{The principle behind Kovacevic method}

Standardized solution of Fe-EDTA complex interacts with hydrogen peroxide by a Fenton-type reaction, leading to the formation of hydroxyl radicals $(\bullet \mathrm{OH})$. These ROS dissolute benzoate, leading to liberation of thiobarbituric acid reactive substances (TBARS). Antioxidants from the added sample of saliva cause suppression of the production of TBARS [10]. Analysis of this reaction is done spectrophotometrically [11].

\section{Statistical analysis}

Data were compiled in an Excel sheet and analyzed using software IBM SPSS Statistics for Windows, Version 22.0. Armonk, NY: IBM Corp. Mean and the standard deviation was calculated and presented in Table 1. TAOC was compared among the Group I (healthy periodontium) and Group II (CP) using unpaired students t-test. $\mathrm{p}<0.05$ was considered statistically significant.

\section{RESULTS}

A study was conducted among 10 subjects with healthy periodontium and 10 patients diagnosed with $\mathrm{CP}$ to assess salivary TAOC

\section{Biochemical assessment of salivary TAOC}

Table 1 and Fig. 1 show the intergroup comparison among Group I and Group II. The mean salivary TAOC was higher in Group I (clinically healthy periodontium) $(796.10 \pm 46.49)$ compared to Group II (CP) (512.05 \pm 50.28$)$, and it differs significantly ( $p<0.001)$ (Fig. 1).

\section{DISCUSSION}

$\mathrm{CP}$ is caused by various hyper-responsive and destructive products of immune response stimulated by microbial plaque around the gingival margin. Inability to maintain the balance between oxidative stress and antioxidant levels makes patients more susceptible to periodontal disease [1]. The major reason for depletion of antioxidant levels in the periodontal disease is due to excessive production of ROS such as $\mathrm{H}_{2} \mathrm{O}_{2}$ and $\mathrm{OH}$ - ion by infiltration of hyperactive PMN leading to the destruction of the connective tissue of periodontal ligament and bone $[12,13]$. Presence of periodontal inflammation is said to be linked with systemic oxidative stress, which, in turn, causes systemic inflammation.

The present study attempts to estimate levels of TAOC in saliva in patients suffering from $\mathrm{CP}$ and compares it with subjects with healthy periodontium. A sample size of 10 subjects with clinically healthy periodontium and 10 subjects with $\mathrm{CP}$ were subjected to salivary estimation of TAOC using Kovacevic method [10]

Baltacıoglu et al. [14] found that both lipid peroxidation levels and TAOC in saliva and serum is reduced in patients with CP while AhmadiMotamayel et al. [15] reported slightly lower TAOC in the periodontitis group compared to healthy control but was not statistically significant. A study was done by Miricescu et al. [16] reported that the salivary antioxidant activity was reduced significantly in CP patients $(\mathrm{p}<0.05)$, which is in an accordance with our study. Results of our study showed higher levels of salivary TAOC in Group I (subjects with clinically healthy periodontium) compare to Group II (subjects with CP). It shows that the oxidative stress in the body is effectively counteracted by the antioxidants. Lower levels of antioxidants in CP direct toward the fact that the disease process is a result of an imbalance between free radical production and antioxidant levels, with the failure of antioxidants to balance the oxidant levels.

Kim et al. [17] found that the salivary total antioxidant level was higher in severe CP patients than in the healthy or gingivitis controls before scaling and root planning, which was in contrast to our present study. Brock et al. [7] found that the salivary antioxidant capacity was lower with periodontitis, but this difference was not significant. Moore et al. [18] reported in their study that salivary TAOC was not statistically different between cases and controls. Wei et al. [19] also showed higher levels of lipid peroxidation levels, total oxidant status

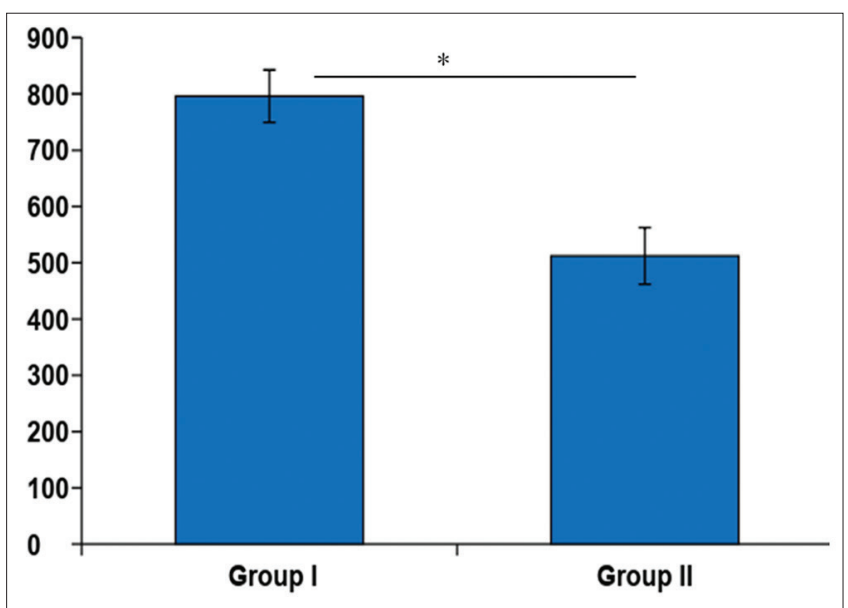

Fig. 1: Comparison of salivary total antioxidant capacity between Groups 1 and 2. Group 1: Subjects with healthy periodontium who showed no signs of periodontal disease. Group 2: Subjects with

CP as defined according to American Academy of Periodontology. Bar indicates the standard deviation within the group. ${ }^{*}$ Statistical significance with Wilcoxon signed-rank test is $\mathrm{p}<0.01$

Table 1: Comparison of salivary TAOC of between Group I and Group II

\begin{tabular}{lllllll}
\hline Group & $\boldsymbol{n}$ & Mean & SD & Mean difference & p-value & 95\% Confidence interval of the difference \\
\hline I & 10 & 796.1 & 46.491 & 284.05 & $<0.001 *$ & $(238.5519,329.5481)$ \\
II & 10 & 512.05 & 50.284 & & & \\
\hline *Statistically significant, SD: Standard deviation. TAOC: Total antioxidant capacity
\end{tabular}


and superoxide dismutase in serum, saliva and gingival crevicular fluid in CP patients. The differences in the results of TAOC among different studies could be explained by different analytical methods used by TAOC assessment $[11,20]$

We evaluated salivary TAOC as they furnish information on the biological interactions among individual antioxidants. It is also more advantageous to estimate TAOC than specific antioxidant level as some of them might be difficult to assay, are expensive, and timeconsuming [21]

In the present study, we estimated TAOC in whole unstimulated saliva because it contains major salivary gland composition, saliva may constitute the first line of defense against free radical-mediated oxidative stress, and in addition, it also has elements of gingival crevicular fluid. It is easily available, non-invasive diagnostic medium for detection of TAOC.

The result of the present study confirms our primary hypothesis that reduction in antioxidant capacity accentuates susceptibility to oxidative stress, and the resulting damage is thought to be involved in the pathogenesis of CP [22]

Within the limitation of our study which had the smaller sample size, we were able to find a statistically significant difference in salivary TAOC in healthy controls and CP. Estimation of oxidative stress and salivary TAOC levels in terms of severity of CP might be more helpful in the thorough understanding of ROS versus antioxidant role in the pathogenesis of CP. Long-term studies with a larger sample size are required to explicate the role played by antioxidants in the progression of periodontal disease.

\section{CONCLUSION}

The result of the current study shows lower levels of salivary TAOC in $\mathrm{CP}$ compared to healthy periodontium. Reduction in TAOC of saliva in the progression of periodontal destruction provides possibilities in future to evolve novel antioxidant treatment strategies to overcome the burden of the free radicals and to play a possible role as a host modulating agent in CP.

\section{AUTHOR'S CONTRIBUTIONS}

All authors have made substantial contributions to the work reported in the manuscript. Raghavendra $U$ and Anupama Rao: Concepting of the study, data collection, and data analysis. Anupama Rao, Jyothi D'esoza, Vinita Ramanath Pai, Sindhu Nair, and Vijaya Kumar: Lab experiment, interpretation, and revision of the article, Bhuvanesh Sukhlal Kalal, and Anupama Rao: Drafting the article and revising the article. All of the authors read and approved the final manuscript of the study to be published.

\section{CONFLICTS OF INTEREST}

The authors declare that there are no conflicts of interest.

\section{REFERENCES}

1. Newman MG, Takei HH, Klokkevold PR, Carranza FA. Carranza's Clinical Periodontology. A South Asia $11^{\text {th }}$ ed. Philadelphia, PA, USA: Elsevier; 2012
2. Van Dyke TE. Cellular and molecular susceptibility determinants for periodontitis. Periodontol 2000 2007;45:10-3.

3. Birben E, Sahiner UM, Sackesen C, Erzurum S, Kalayci O. Oxidative stress and antioxidant defense. World Allergy Organ J 2012;5:9-19.

4. Sculley DV, Langley-Evans SC. Periodontal disease is associated with lower antioxidant capacity in whole saliva and evidence of increased protein oxidation. Clin Sci Lond 2003;105:167-72.

5. Akalin FA, Toklu E, Renda N. Analysis of superoxide dismutase activity levels in gingiva and gingival crevicular fluid in patients with chronic periodontitis and periodontally healthy controls. J Clin Periodontol 2005;32:238-43.

6. Lutfioğlu M, Aydoğdu A, Atabay VE, Sakallioğlu EE, Avci B. Gingival crevicular fluid oxidative stress level in patients with periodontal disease and hyperlipidemia. Braz Oral Res 2017;31:e110.

7. Brock GR, Butterworth CJ, Matthews JB, Chapple IL. Local and systemic total antioxidant capacity in periodontitis and health. J Clin Periodontol 2004;31:515-21.

8. Armitage GC. Development of a classification system for periodontal diseases and conditions. Ann Periodontol 1999;4:1-6.

9. Raghavendra U, Rao A, Kashyap RS, D’Souza J, Kumar V, Kalal BS, et al. Vitamin C supplementation as an adjunct to non-surgical therapy in the treatment of chronic periodontitis- a clinical and biochemical study. J Int Oral Health 2018;10. http://dx.doi.org/10.4103/jioh. jioh_158_18 (In Press).

10. Koracevic D, Koracevic G, Djordjevic V, Andrejevic S, Cosic V. Method for the measurement of antioxidant activity in human fluids. J Clin Pathol 2001;54:356-61.

11. Dontha S. A review on antioxidant methods. Asian J Pharm Clin Res 2016;9:14-32.

12. Canakçi CF, Ciçek Y, Canakçi V. Reactive oxygen species and human inflammatory periodontal diseases. Biochemistry (Mosc) 2005;70:619-28

13. Bhat GH, Kalal BS, Prasad G. Serum amylase or serum lipase, a comparison in acute pancreatitis. Turk J Biochem 2018;43. http:// dx.doi.org/10.1515/tjb-2017-0318 (In Press)

14. Baltacıoğlu E, Yuva P, Aydın G, Alver A, Kahraman C, Karabulut E, et al. Lipid peroxidation levels and total oxidant/antioxidant status in serum and saliva from patients with chronic and aggressive periodontitis. Oxidative stress index: A new biomarker for periodontal disease? J Periodontol 2014;85:1432-41

15. Ahmadi-Motamayel F, Goodarzi MT, Jamshidi Z, Kebriaei R. Evaluation of salivary and serum antioxidant and oxidative stress statuses in patients with chronic periodontitis: A Case-control study. Front Physiol 2017;8:189.

16. Miricescu D, Totan A, Calenic B, Mocanu B, Didilescu A, Mohora M, et al. Salivary biomarkers: Relationship between oxidative stress and alveolar bone loss in chronic periodontitis. Acta Odontol Scand 2014;72:42-7.

17. Kim SC, Kim OS, Kim OJ, Kim YJ, Chung HJ. Antioxidant profile of whole saliva after scaling and root planing in periodontal disease. J Periodontal Implant Sci 2010;40:164-71.

18. Moore S, Calder KA, Miller NJ, Rice-Evans CA. Antioxidant activity of saliva and periodontal disease. Free Radic Res 1994;21:417-25.

19. Wei D, Zhang XL, Wang YZ, Yang CX, Chen G. Lipid peroxidation levels, total oxidant status and superoxide dismutase in serum, saliva and gingival crevicular fluid in chronic periodontitis patients before and after periodontal therapy. Aust Dent J 2010;55:70-8.

20. Wang J, Schipper HM, Velly AM, Mohit S, Gornitsky M. Salivary biomarkers of oxidative stress: A critical review. Free Radic Biol Med 2015;85:95-104

21. Maxwell SR, Dietrich T, Chapple IL. Prediction of serum total antioxidant activity from the concentration of individual serum antioxidants. Clin Chim Acta 2006;372:188-94.

22. Pendyala G, Thomas B, Kumari S. The challenge of antioxidants to free radicals in periodontitis. J Indian Soc Periodontol 2008;12:79-83. 